\title{
Fixed-wing UAV guidance law for ground target over-flight tracking
}

\author{
ZHANG Min*, ZHENG Chenming, and HUANG Kun \\ College of Automation Engineering, Nanjing University of Aeronautics and Astronautics, Nanjing 211106, China
}

\begin{abstract}
This paper studies the problem of tracking a ground target for a fixed-wing unmanned aerial vehicle (UAV) based on the proposed guidance law. The algorithm ensures that a UAV continuously overflies the target whether it is fixed or moving. The requirements of the UAV flight constraints such as bounded airspeed and acceleration are considered. A Lyapunov function is constructed to prove the stability of the proposed guidance law, and parameter design criteria have been developed. Considering the fixed and moving ground targets, numerical simulations are performed to verify the feasibility and benefits of the proposed guidance algorithm.
\end{abstract}

Keywords: unmanned aerial vehicle (UAV), ground target, guidance law, over-flight, stability.

DOI: $10.21629 /$ JSEE.2019.02.16

\section{Introduction}

During the past decade, rapid developments have been witnessed in the unmanned aerial vehicle (UAV), owing to its large potential applications [1-5] in both military and civilian domains such as surveillance, reconnaissance and monitoring. For these applications, it is a challenging task for the UAV to track a ground target.

The UAV has a small field of view in general, so it is a vital part of the target tracking system that the motion control of the UAV becomes. Meanwhile, compared with the rotary-wing UAV, the flight constraints, such as minimum/maximum speed and minimum turning radius on the fixed-wing UAV, make the corresponding solution strategies complicated.

There exist two kinds of tracking modes for a UAV to track a ground target: standoff tracking [6-11] and over-flight tracking [12-17]. It is an advantage of standoff tracking that it can decrease the risk of exposure [18-21]. However, considering the limitation of the minimum tur-

Manuscript received December 29, 2017.

*Corresponding author.

This work was supported by the Aeronautical Science Foundation of China (20160152001). ning radius of the $\mathrm{UAV}$, this tracking method is only suited to tracking slow targets [22-24]. According to [24], for the standoff tracking mode, the ground target velocity cannot be over 30 percent of the maximum cruising speed of the UAV, while the over-flight tracking mode has no limit.

When the ground target is moving at a changing speed, the UAV speed is also changing in most of the existing studies [25-28]. Although this method reduces the design difficulty, there are still some problems.

Firstly, wide and frequent speed regulation may cause instability of UAV altitude; even if we have high performance of speed-altitude control loop, short-term longitudinal coupling vibration cannot be avoided [29-32].

Secondly, when the target velocity is less than a certain value, it is possible to cause the UAV to stall. The UAV must switch to the hover mode in such a situation.

Finally, when multiple UAVs are used to track a ground target in formation [33-35], a more reasonable option is to use the speed control channel for a small range adjustment of the horizontal space between the UAVs.

In [13] and [14], a guidance law based on line of sight was given, in which the trajectory of the UAV was composed of straight line and arc. When the relative distance between the UAV and the target is less than a certain threshold, the trajectory of the UAV is a straight line, and the over-flight is achieved in this condition. When the relative distance is greater than the threshold, the trajectory of the UAV is switched from the straight line to the arc, so this trajectory can be periodically switched between the straight line and the arc. Obviously, the deficiency of this guidance law is that it can only be used in the form of a piecewise function, and the stability of the system cannot be proved in a unified framework.

While in [23], a kind of indirect over-flight tracking method was given, the trajectory of the UAV was a lemniscates curve. First, a virtual target is created based on the trajectory of the real target, and then we design a guid- 
ance law to track the virtual target. The disadvantage of this method is that the design process is too complicated.

This paper presents a new tracking method for the fixedwing UAV to track a ground target, and over-flight tracking of fixed target and moving target is achieved by using the new guidance law. The proposed method is a direct design method. Compared with the existing methods, the new guidance law is simpler, does not need any piecewise functions, and Lyapunov stability proof is completed within a unified framework.

\section{Problem statement}

The flight control system of a UAV usually consists of the stabilization loop (inner loop) and the guidance loop (outer loop). In this paper, we assume that the inner loop has been designed, and it can well respond to instructions that are sent by the outer loop. The UAV is considered at a constant altitude, which can be simplified as a two dimensional guidance problem.

The system dynamics can be described by the following kinematical model:

$$
\left\{\begin{array}{l}
\dot{x}=V \cos \psi+W_{x} \\
\dot{y}=V \sin \psi+W_{y} \\
\dot{\psi}=\omega
\end{array}\right.
$$

where $[x, y]^{\mathrm{T}}$ denotes the two dimensional location of the UAV, $V$ denotes the UAV speed, $\psi$ denotes the heading angle, and $W_{x}, W_{y}$ are the velocity components of wind on the $x$ and $y$ axes. $\omega$ is the control input to be designed. $\left[x_{t}, y_{t}\right]^{\mathrm{T}}$ denotes the two dimensional location of the target, then the range between the UAV and the target is shown as follows:

$$
\rho=\sqrt{\left(x-x_{t}\right)^{2}+\left(y-y_{t}\right)^{2}} .
$$

For the sake of representing the relative motion between the UAV and the ground target, according to Fig. 1, (1) can be rewritten as

$$
\left\{\begin{array}{l}
\dot{\rho}=V_{t} \cos \left(\theta+\psi-\psi_{t}\right)-V_{g} \cos \theta \\
\dot{\theta}=\frac{-V_{t} \sin \left(\theta+\psi-\psi_{t}\right)+V_{g} \sin \theta}{\rho}-\dot{\psi}
\end{array}\right.
$$

where $\theta$ denotes the angle between $V$ and $\rho, \psi_{t}$ is the angle of the target speed, and $\sigma$ is the angle between $\rho$ and the North.

$$
\begin{gathered}
\theta=\sigma-\psi, \\
V_{g}=\sqrt{\left(V \cos \psi+W_{x}\right)^{2}+\left(V \sin \psi+W_{y}\right)^{2}} .
\end{gathered}
$$

When there is no wind and the ground target is fixed, (2) can be rewritten as

$$
\left\{\begin{array}{l}
\dot{\rho}=-V \cos \theta \\
\dot{\theta}=\frac{V \sin \theta}{\rho}-\omega
\end{array} .\right.
$$

In (3), the state variables are transformed from $[x, y, \psi]^{\mathrm{T}}$ to $[\rho, \theta]^{\mathrm{T}}$. It is known that if the velocity of the UAV is constant, $\rho$ and $\theta$ can be determined by each other.

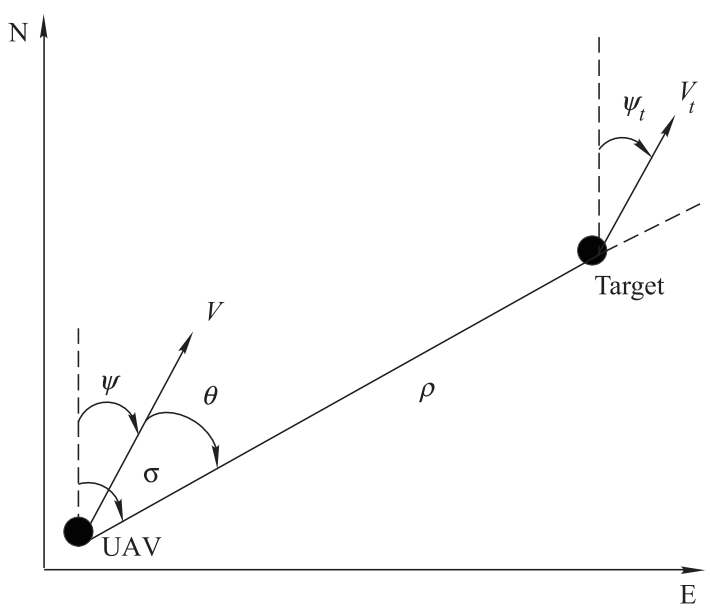

Fig. 1 Geometry of tracking ground target

\section{Guidance law design}

In the process of over-flight tracking, the UAV should change its heading angle under the guidance law, that is, the horizontal acceleration is perpendicular to the direction of the UAV speed while the UAV flies over the ground target.

In this paper, a new guidance law based on the line of sight is proposed as

$$
\omega=\frac{a_{n}}{V}=\frac{k_{1} \theta}{V\left(\cosh \theta-k_{2}\right)} .
$$

In (4), $k_{1}$ and $k_{2}$ are constant gains, and $k_{1}>0, k_{2}>0$. Compared with the guidance laws of (9) in [13], (9) in [14], (48) in [15], (6.99) in [16] and (4) in [17], this new guidance law is the most compact one, which has no piecewise functions. A simple guidance law is more conducive to engineering practice. In Section 6.4, we can see that the guidance law proposed in this paper also has advantages in average distance between the UAV and the target.

For dynamics of the UAV in (3) subject to the guidance law in (4), the close-loop system can be given by

$$
\begin{gathered}
\dot{\rho}=-V \cos \theta, \\
\dot{\theta}=\frac{V \sin \theta}{\rho}-\frac{k_{1} \theta}{V\left(\cosh \theta-k_{2}\right)} .
\end{gathered}
$$

\section{Stability analysis of tracking fixed target}

The stability of the closed loop system in (5) will be analyzed and proved as in follows. 
Theorem 1 Consider the UAV dynamics in (3) subject to the guidance law in (4), and set $t_{0} \geqslant 0$, for any $\theta\left(t_{0}\right) \in[0,2 \pi)$. If $0<k_{2}<1$, then there exists $t_{1} \geqslant t_{0}$ such that $\theta\left(t_{1}\right) \in[0, \pi]$, and for any $t \geqslant t_{1}, \theta(t) \in[0, \pi]$.

Proof The proof can be divided into two steps. We firstly prove the existence of time $t_{1} \geqslant t_{0}$, which makes $\theta\left(t_{1}\right) \in[0, \pi]$.

When $\theta\left(t_{0}\right) \in(\pi, 2 \pi), \sin \left(\theta\left(t_{0}\right)\right)<0$, since $\cosh \left(\theta\left(t_{0}\right)\right)-k_{2}>0$, according to (5b), we know that $\dot{\theta}\left(t_{0}\right)<0$. In the same way, for any $t_{0}+\Delta t \in(\pi, 2 \pi)$, where $t$ is any positive constant, there always is $\dot{\theta}\left(t_{0}+\right.$ $\Delta t)<0$. Thus after a period of time, there exists $t_{1}=$ $t_{0}+\Delta t$ such that $\theta\left(t_{1}\right) \in[0, \pi]$.

Next, we prove that for any $t \geqslant t_{1}, \theta(t) \in[0, \pi]$.

According to (5b), when $\theta\left(t_{1}\right)=0, \dot{\theta}\left(t_{1}\right)=0$, we know that $a_{n}=0, \dot{\rho}=-V$, that is, the UAV will fly over the target in straight line motion. Then $\dot{\rho}>0$ and $\theta(t)=\pi$ happen, and we can obtain $\dot{\theta}(t)<0$. $\theta(t)$ will monotonously decrease, so $\theta(t) \in[0, \pi]$ holds for any $t \geqslant t_{1}$.

By combining the above analysis, there exists $t_{1} \geqslant t_{0}$ such that $\theta\left(t_{1}\right) \in[0, \pi]$, and for any $t \geqslant t_{1}, \theta(t) \in[0, \pi]$.

Let $\dot{\rho}=0, \dot{\theta}=0$ in (5), we can obtain that $\theta_{e 1}=\frac{\pi}{2}$, $\theta_{e 2}=\frac{3 \pi}{2}, \rho_{e}=\frac{V^{2}\left(\cosh \theta_{e}-k_{2}\right)}{k_{1} \theta_{e}} \cdot\left[\rho_{e}, \theta_{e 1}\right]^{\mathrm{T}},\left[\rho_{e}, \theta_{e 2}\right]^{\mathrm{T}}$ are two equilibrium points of the system. According to Theorem $1,\left[\rho_{e}, \theta_{e 1}\right]^{\mathrm{T}}$ is the equilibrium point to be considered.

According to the above mentioned contents, ranges of $k_{1}$ and $k_{2}$ have been given. While, by considering a UAV flying along constant rate turn maneuver, the lateral acceleration can be written as

$$
a_{n}=\frac{V^{2}}{R}
$$

where $R$ denotes the radius of circular motion of the UAV.

Therefore, the lateral acceleration of the UAV is constrained by the minimum turning radius $R_{\min }$. Moreover, consider the flight equation for the no sideslip turn:

$$
\dot{\psi}=\frac{g}{V} \tan \phi
$$

where $\phi$ is the bank angle and $g$ is the gravity acceleration. We can obtain

$$
R_{\min }=\frac{V^{2}}{g \tan \phi_{\max }}
$$

where $\phi_{\max }$ is the maximum bank angle.

According to the following inequalities:

$$
\left\{\begin{array}{l}
\rho_{e} \geqslant R_{\min } \\
a_{n_{\max }} \leqslant g \tan \phi_{\max }
\end{array}\right.
$$

$k_{1}$ and $k_{2}$ should satisfy the following equation:

$$
k_{1} \leqslant \frac{g \tan \phi_{\max }\left(\cosh \theta_{e}-k_{2}\right)}{\theta_{e}} .
$$

Furthermore, in the design of guidance parameters $k_{1}$ and $k_{2}$, we also need to consider the requirements of the rapidity of the guidance loop and the bandwidth of the roll loop as well.

Theorem 2 For fixed ground target, considering the UAV dynamics in (3) subject to the guidance law in (4), the closed loop system in (5) is asymptotically stable.

Proof In order to prove the asymptotic stability, (5) can be rewritten as

$$
\left[\begin{array}{l}
\dot{\rho} \\
\dot{\theta}
\end{array}\right]=\left[\begin{array}{cc}
0 & -m \\
m & -1
\end{array}\right] \cdot\left[\begin{array}{l}
\rho \\
\theta
\end{array}\right]+\left[\begin{array}{c}
-V \cos \theta+m \theta \\
\frac{V \sin \theta}{\rho}-\frac{a_{n}}{V}-m \rho+\theta
\end{array}\right]
$$

where $m$ is a constant value and $m>0$.

Let

$$
\boldsymbol{A}_{0}=\left[\begin{array}{cc}
0 & -m \\
m & -1
\end{array}\right] \text {. }
$$

Obviously, $\boldsymbol{A}_{0}$ is a Hurwitz matrix.

And let

$$
f(\boldsymbol{x})=\left[\begin{array}{c}
-V \cos \theta+m \theta \\
\frac{V \sin \theta}{\rho}-\frac{a_{n}}{V}-m \rho+\theta
\end{array}\right]
$$

where $\boldsymbol{x}=[\rho, \theta]^{\mathrm{T}}$, then (11) can be rewritten as

$$
\dot{\boldsymbol{x}}=\boldsymbol{A}_{0} \boldsymbol{x}+f(\boldsymbol{x}) .
$$

Now we can define a candidate Lyapunov function:

$$
L(\boldsymbol{x})=\boldsymbol{x}^{\mathrm{T}} \boldsymbol{P} \boldsymbol{x}
$$

where $\boldsymbol{P}$ is a symmetric positive definite matrix. Since $\boldsymbol{A}_{0}$ is a Hurwitz matrix, we can obtain that for any symmetric positive definite matrix $Q$, there exists a symmetric positive definite matrix $\boldsymbol{P}$ such that

$$
\boldsymbol{A}_{0}^{\mathrm{T}} \boldsymbol{P}+\boldsymbol{P} \boldsymbol{A}_{0}=-\boldsymbol{Q} .
$$

It can be verified that $L(\boldsymbol{x}) \geqslant 0$, and $L(\boldsymbol{x})=0$ only at $\|\boldsymbol{x}\|=0$.

The derivative of (15) yields

$$
\begin{gathered}
\dot{L}(\boldsymbol{x})=\dot{\boldsymbol{x}}^{\mathrm{T}} \boldsymbol{P} \boldsymbol{x}+\boldsymbol{x}^{\mathrm{T}} \boldsymbol{P} \dot{\boldsymbol{x}}= \\
{\left[\boldsymbol{x}^{\mathrm{T}} \boldsymbol{A}_{0}^{\mathrm{T}}+f(\boldsymbol{x})^{\mathrm{T}}\right] \boldsymbol{P} \boldsymbol{x}+\boldsymbol{x}^{\mathrm{T}} \boldsymbol{P}\left[\boldsymbol{A}_{0} \boldsymbol{x}+f(\boldsymbol{x})\right]=} \\
\boldsymbol{x}^{\mathrm{T}}\left(\boldsymbol{A}_{0}^{\mathrm{T}} \boldsymbol{P}+\boldsymbol{P} \boldsymbol{A}_{0}\right) \boldsymbol{x}+2 f(\boldsymbol{x})^{\mathrm{T}} \boldsymbol{P} \boldsymbol{x} .
\end{gathered}
$$

Substituting (16) into (17) yields

$$
\dot{L}(\boldsymbol{x})=-\boldsymbol{x}^{\mathrm{T}} \boldsymbol{Q} \boldsymbol{x}+2 f(\boldsymbol{x})^{\mathrm{T}} \boldsymbol{P} \boldsymbol{x} .
$$


Since $\theta \in[0, \pi],-\cos \theta \leqslant|\theta|, \sin \theta \leqslant|\theta|$ and $\cosh \theta \geqslant 1$, we can obtain

$$
|\theta| \sqrt{(V+m)^{2}+\left(V+\frac{k_{1}}{V\left(1-k_{2}\right)}+(m+1)\right)^{2}} .
$$

Let

$$
\alpha=\sqrt{\left[(V+m)^{2}+\left(V+\frac{k_{1}}{V\left(1-k_{2}\right)}+(m+1)\right)^{2}\right]} .
$$

Inequality (19) can be rewritten as

$$
\|f(\boldsymbol{x})\| \leqslant \alpha|\theta| .
$$

Since $\|\boldsymbol{x}\| \geqslant|\theta|$, it results

$$
\|f(\boldsymbol{x})\| \leqslant \alpha\|x\| .
$$

At last, since $\boldsymbol{x}^{\mathrm{T}} \boldsymbol{Q} \boldsymbol{x} \geqslant \lambda_{\min }(\boldsymbol{Q})$, we can obtain

$$
\dot{L}(\boldsymbol{x})<-\left(\lambda_{\min }(\boldsymbol{Q})-2 \alpha\|\boldsymbol{P}\|\right)\|\boldsymbol{x}\|^{2} .
$$

If

$$
\|\boldsymbol{P}\|<\frac{\lambda_{\min }(\boldsymbol{Q})}{2 \alpha}
$$

then the derivative function $\dot{L}(\boldsymbol{x})<0$.

For example, let $\boldsymbol{Q}=\boldsymbol{I}$, according to (16), we can obtain

$$
\boldsymbol{P}=\left[\begin{array}{cc}
1+\frac{1}{2 m^{2}} & -\frac{1}{2 m} \\
-\frac{1}{2 m} & 1
\end{array}\right]
$$

According to (20), $\alpha \geqslant \sqrt{2} V$, then according to (25), we can obtain

$$
1+\frac{1}{4 m^{2}} \leqslant \frac{1}{2 \sqrt{2} V} .
$$

Obviously, variable $m$ that satisfies the above inequality exists, and the closed loop system (5) is asymptotically stable.

\section{Stability analysis of tracking moving target}

When the ground target is moving, the UAV dynamics in (3) can be extended in the following equations:

$$
\left\{\begin{array}{l}
\dot{\rho}=V_{t} \cos \left(\theta+\psi-\psi_{t}\right)-V \cos \theta \\
\dot{\theta}=\frac{1}{\rho}\left(-V_{t} \sin \left(\theta+\psi-\psi_{t}\right)\right)+\frac{V \sin \theta}{\rho}-\omega
\end{array} .\right.
$$

Substituting (4) into (27) yields

$$
\left\{\begin{array}{c}
\dot{\rho}=V_{t} \cos \left(\theta+\psi-\psi_{t}\right)-V \cos \theta \\
\dot{\theta}=\frac{1}{\rho}\left(-V_{t} \sin \left(\theta+\psi-\psi_{t}\right)\right)+ \\
\frac{V \sin \theta}{\rho}-\frac{k_{1} \theta}{V\left(\cosh \theta-k_{2}\right)}
\end{array} .\right.
$$

The stability of the closed loop system (28) will be analyzed and proved in the following.

Theorem 3 For moving ground targets, considering the UAV dynamics in (27) subject to the guidance law in (4), the closed loop system in (28) is ultimate boundedness stable.

Proof Similar to the derivation of Theorem 2, (28) can be rewritten as

$$
\dot{\boldsymbol{x}}=\boldsymbol{A}_{0}(\boldsymbol{x})+g(\boldsymbol{x}) .
$$

Let $\psi_{r}=\psi-\psi_{t}$, it results

$$
g(\boldsymbol{x})=\left[\begin{array}{l}
V_{t} \cos \left(\theta+\psi_{r}\right)+m \theta \\
-\frac{V_{t} \sin \left(\theta+\psi_{r}\right)}{\rho}-m \rho+\theta
\end{array}\right] .
$$

Then we can obtain

$$
\|g(\boldsymbol{x})\| \leqslant \alpha\|\boldsymbol{x}\|+\sqrt{2} V_{t} .
$$

Let $V_{t \max }$ be the maximum velocity value, by combining the above analysis, we can obtain

$$
\begin{gathered}
\dot{L}(\boldsymbol{x})<-\|\boldsymbol{x}\| . \\
{\left[\left(\lambda_{\min }(\boldsymbol{Q})-2 \alpha\|\boldsymbol{P}\|\right)\|\boldsymbol{x}\|-2 \sqrt{2} V_{t \max }\|\boldsymbol{P}\|\right] .}
\end{gathered}
$$

Now if

$$
\|\boldsymbol{x}\|>\frac{2 \sqrt{2} V_{t \max }\|\boldsymbol{P}\|}{\lambda_{\min }(\boldsymbol{Q})-2 \alpha\|\boldsymbol{P}\|}
$$

then the Lyapunov time derivative function $\dot{L}(\boldsymbol{x})<0$.

Define an open set

$$
\boldsymbol{\Omega}=\left\{\boldsymbol{x}:\|\boldsymbol{x}\|>\frac{2 \sqrt{2} V_{t \max }\|\boldsymbol{P}\|}{\lambda_{\min }(\boldsymbol{Q})-2 \alpha\|\boldsymbol{P}\|}\right\} .
$$

If $\boldsymbol{x} \in \boldsymbol{\Omega}$ and (24) is satisfied, the closed loop system in (28) is ultimate boundedness stable.

\section{Simulation results}

In this section, some simulation results are shown for the sake of demonstrating the effectiveness of the proposed guidance law. In these simulations, the motion of the ground target is considered as stationary, linear with variable speed and circular with constant speed. The velocity of the UAV is set constant at $10 \mathrm{~m} / \mathrm{s}$. When the wind effect is considered, it has a constant from the south and the constant speed equals to $3 \mathrm{~m} / \mathrm{s}$. The gains adopted in the guidance law are $k_{1}=5.5, k_{2}=0.5$. 


\subsection{Fixed target}

With the UAV's initial position $(-100,20)$, and initial heading pointing of the south-east, the simulation continues for $200 \mathrm{~s}$.

In Fig. 2, we consider no wind effect and the UAV can pass over the target. Furthermore, Fig. 3 shows the trajectory of the UAV with wind effect. No matter whether the wind exists, the UAV is able to pass over the target, only trajectories are different.

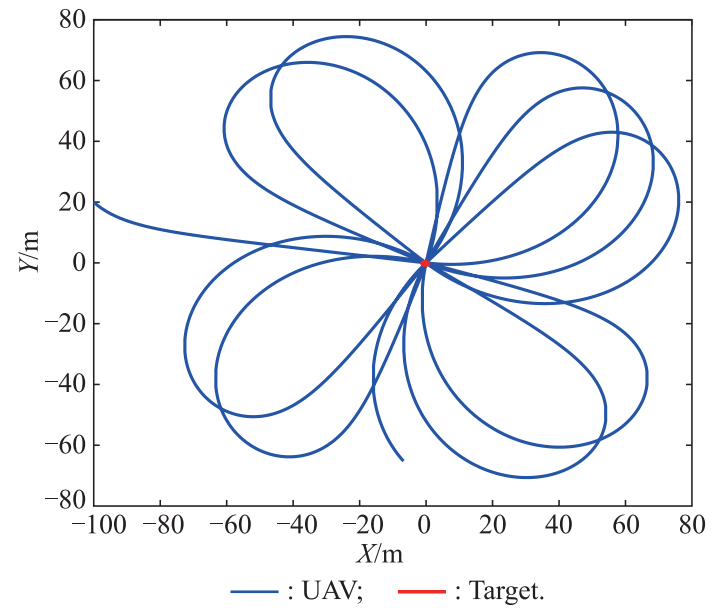

Fig. 2 Trajectory of tracking fixed target with no wind

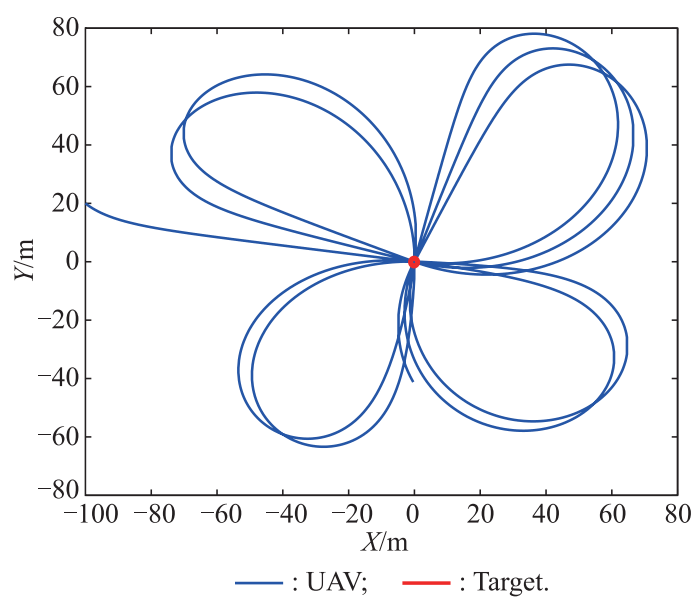

Fig. 3 Trajectory of tracking fixed target with wind effect

In Fig. 4 and Fig. 5, in both cases the distance between the UAV and the target goes periodically to zero. The only slight difference that can be seen between the two figures is the value of distance.

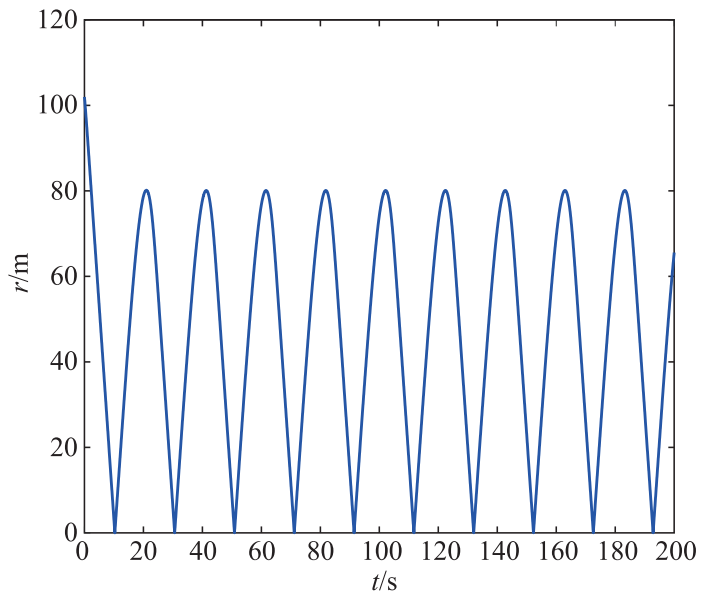

Fig. 4 Relative range of tracking fixed target with no wind

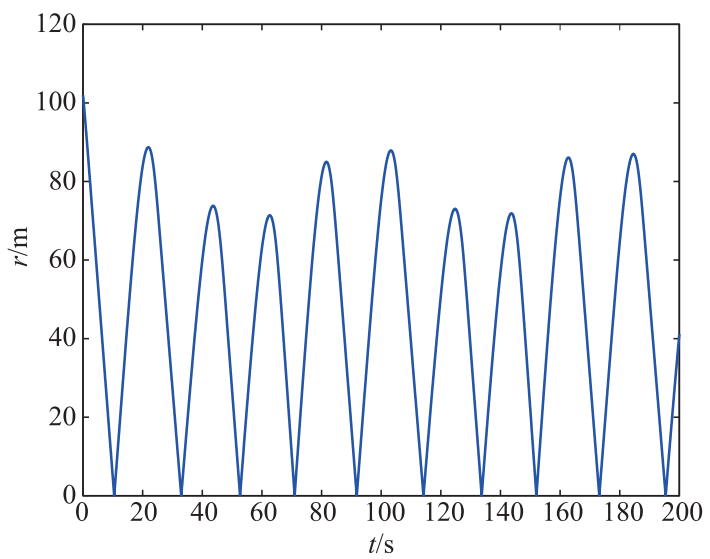

Fig. 5 Relative range of tracking fixed target with wind effect

\subsection{Linear moving target}

Suppose that the target moves along a straight line toward the north east, and the speed of the target is variable but always less than that of the UAV. The target's profile velocity is shown in Fig. 6, and the simulation lasts $250 \mathrm{~s}$.

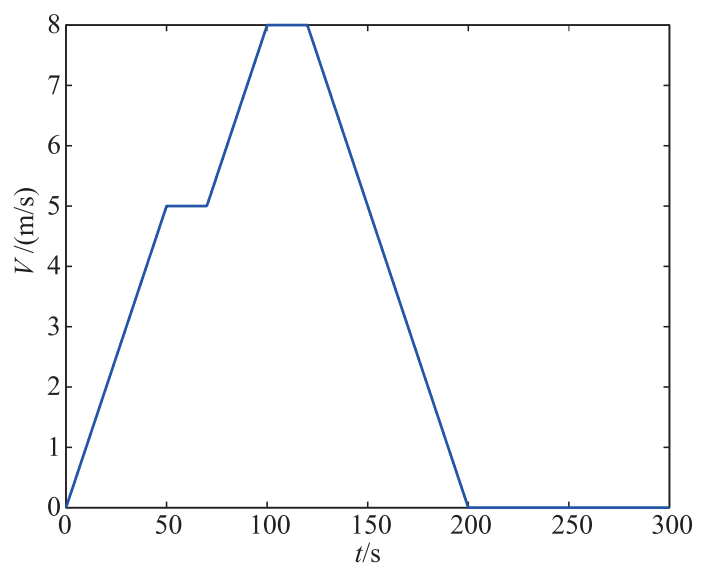

Fig. 6 Target profile velocity 
In Fig. 7 and Fig. 8, it is obvious that the UAV can track the target in both cases. When the target keeps fixed, the UAV flies over it continuously. Due to the presence of the wind, the trajectories generated by the guidance law are different. Fig. 9 and Fig. 10 show the range between the target and the UAV. Once the UAV reaches the target, it starts to loiter around it as in the previous case.

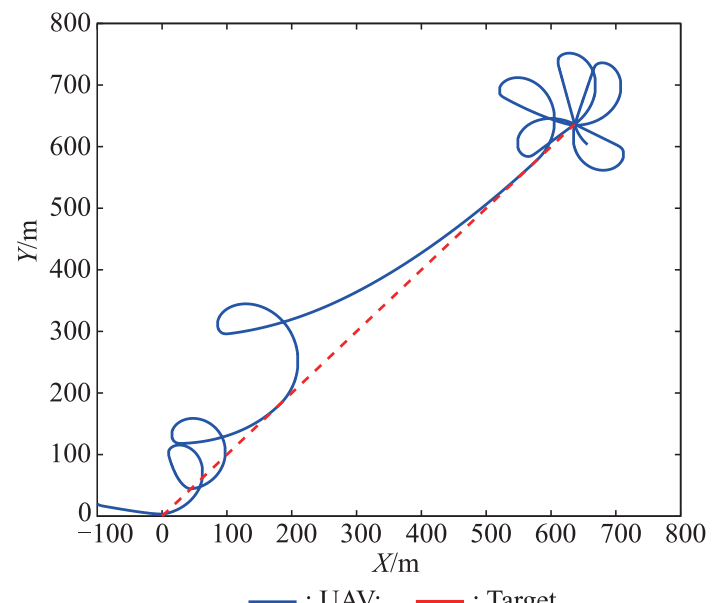

Fig. 7 Trajectory of tracking linear moving target with no wind

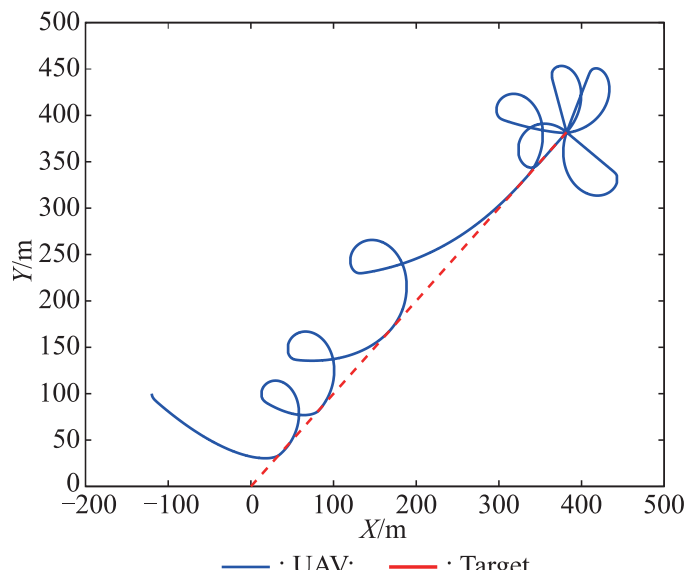

Fig. 8 Trajectory of tracking linear moving target with wind effect

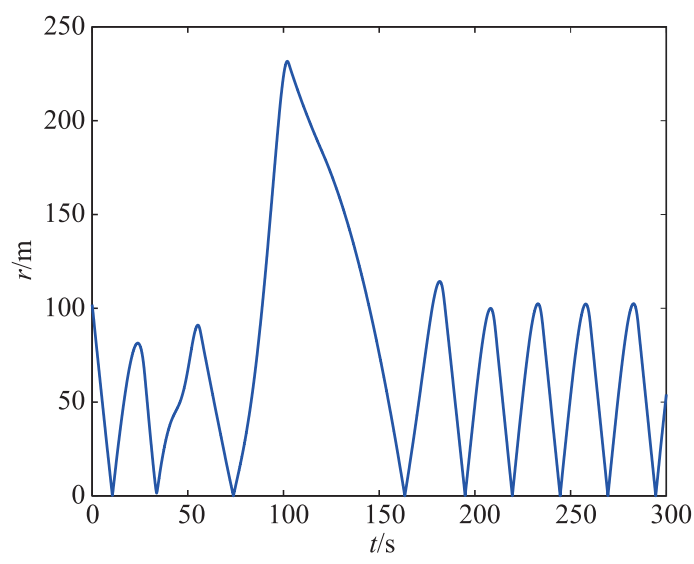

Fig. 9 Relative range of tracking linear moving target with no wind

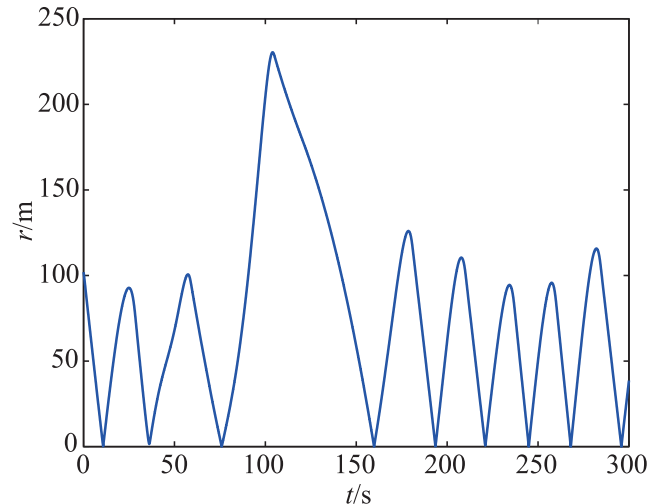

Fig. 10 Relative range of tracking linear moving target with wind effect

\subsection{Circular moving target}

In this case, suppose that the target moves along a circular trajectory. The purpose of this test is to ensure the performance when the target is implementing a bend trajectory. In this case, we suppose that the target velocity and lateral acceleration are constant, set at $5 \mathrm{~m} / \mathrm{s}$ and $0.05 \mathrm{~m} / \mathrm{s}^{2}$, respectively.

It is shown that the UAV is able to track the target both in Fig. 11 and Fig. 12. Fig. 13 shows that the range between the UAV and the target tends to be zero.

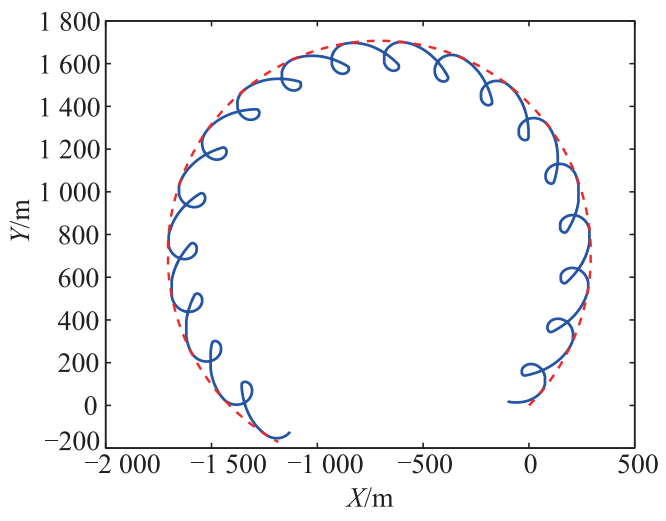

— : UAV; — : Target.

Fig. 11 Trajectory of tracking circular motion target with no wind

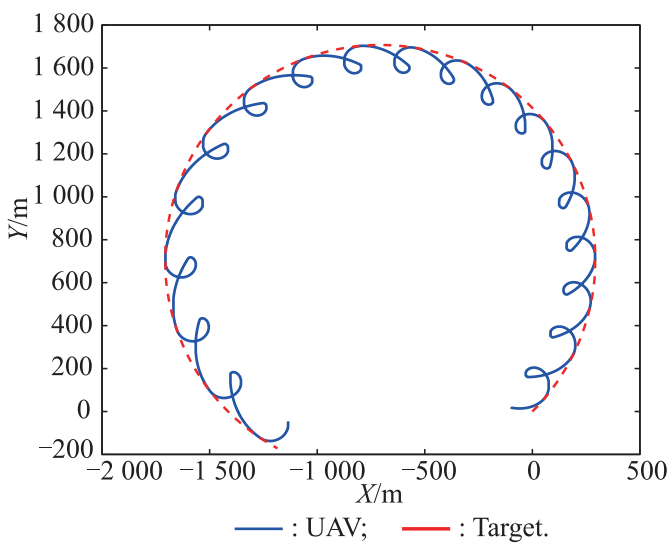

Fig. 12 Trajectory of tracking circular motion target with wind effect 


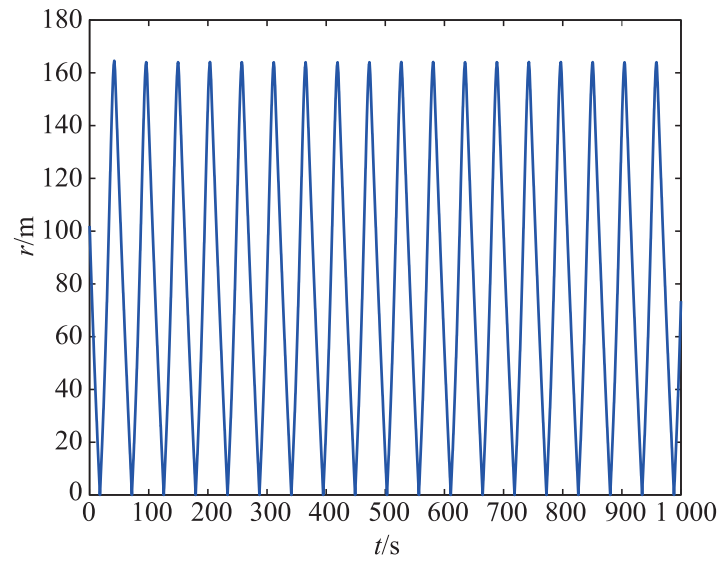

Fig. 13 Relative range of tracking circular motion target with no wind

Note that the UAV is able to reach the target in Fig. 14, and the trajectory is not periodic anymore owing to the wind effect.

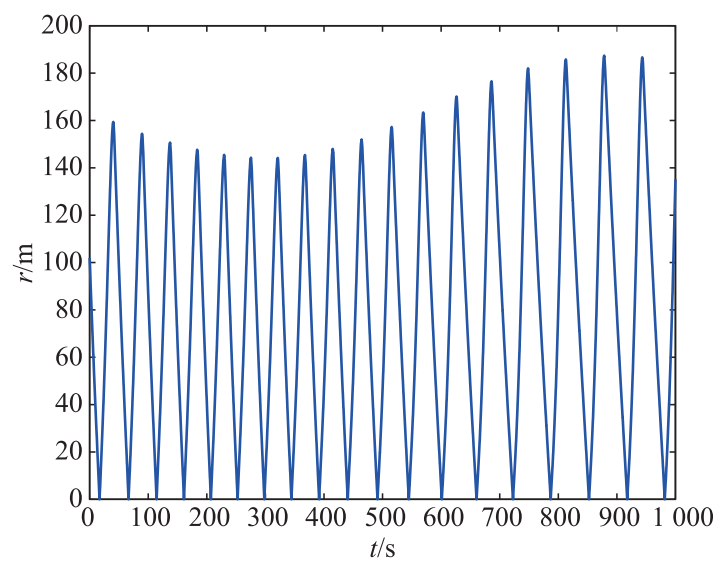

Fig. 14 Relative range of tracking circular motion target with wind effect

\subsection{Tracking performance analysis}

In the above mentioned over-flight tracking guidance laws, the one in [14] is the most representative. In order to evaluate the performance of the guidance law in this paper, a compareison with that is made. The distance between the UAV and the ground target is taken as the criterion.

In this scenario, the target is fixed at the origin. The velocity of the UAV is a constant of $10 \mathrm{~m} / \mathrm{s}$. The guidance law used as a comparison is derived from [14], and its parameters are considered to have been well chosen. The initial position of the UAV is set at $(0,0)$, and the target at $(100$, 100). The simulation results are shown in Fig. 15.

For the guidance of over-flight tracking, the average distance is an important index of tracking performance. The smaller distance means that the observation of the target can be more clear and more continuous. In Fig. 15, it can be known obviously that the guidance law in this paper is closer to approaching the target than that in [14] (solid and dot lines in Fig. 15). The reason for this is that the guidance law in [14] uses a fixed parameter $R_{0}$ which is closely related to the relative distance. When trying to reduce $R_{0}$ for achieving the same tracking performance as the proposed guidance law, the tracking distance diverges (dash line in Fig. 15). Even if other parameters are adjusted, there is no improvement. Therefore, it can be concluded that the tracking performance of the guidance law proposed in this paper is better than that in [14].

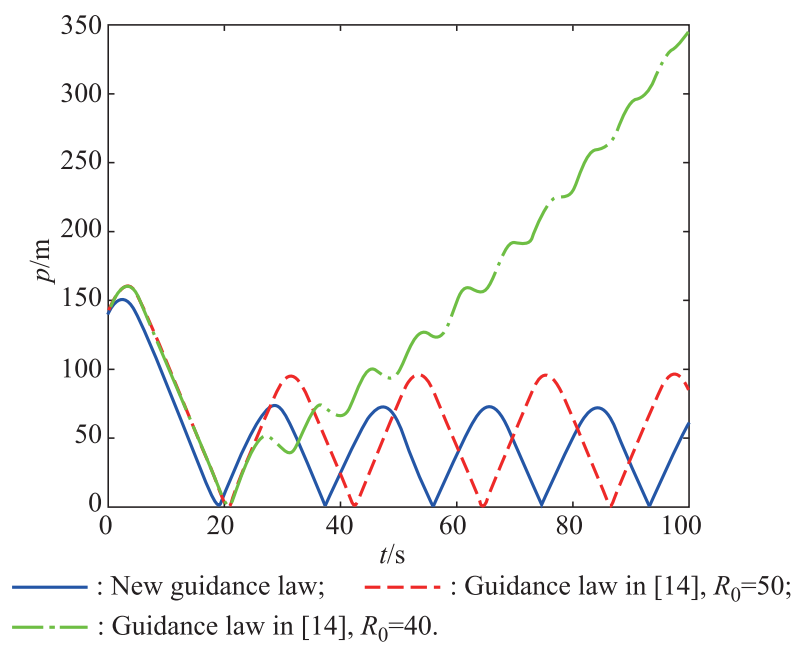

Fig. 15 Comparison of relative range

\section{Conclusions}

In this paper, a new guidance law is proposed for a fixedwing UAV to track a ground target. The guidance law only uses the line of sight signal and satisfies the UAV's flight constraints. Compared with the existing one, the proposed guidance law does not require piecewise functions. A Lyapunov function is constructed to prove the stability of the proposed guidance law. Simulation results are presented to demonstrate the effectiveness in the case of no wind effect or a constant wind.

\section{References}

[1] WANG D B, WANG Y, JIANG W Y, et al. Unmanned aerial vehicles cooperative path planning for ground target tracking via chemical reaction optimization. Scientia Sinica Technologica, 2015, 45(6): 583 -594. (in Chinese)

[2] CHEN Z J, WEI J Z, WANG Y X, et al. UAV autonomous control levels and system structure. Acta Aeronautica et Astronautica Sinica, 2011, 32(6): 1075 - 1083. (in Chinese)

[3] BETTS J T. Survey of numerical methods for trajectory optimization. Journal of Guidance, Control and Dynamics, 1998, 21(2): $193-207$.

[4] CAO Y. UAV circumnavigating an unknown target under a GPS-denied environment with range-only measurements. Automatica, 2015, 55(1): 150-158. 
[5] CAO Y, MUSE J, CASBEER D, et al. Circumnavigation of an unknown target using UAVs with range and range rate measurements. Proc. of the 52nd IEEE Conference on Decision and Control, 2013: 3617-3622.

[6] MATVEEV A S, TEIMOORI H, SAVKIN A V. Rangeonly measurements based target following for wheeled mobile robots. Automatica, 2011, 47(1): $177-184$.

[7] DEGHAT M, SHAMES I, ANDERSON B D O, et al. Localization and circumnavigation of a slowly moving target using bearing measurements. IEEE Trans. on Automatic Control, 2014, 59(8): 2182-2188.

[8] SANGHYUK P. Circling over a target with relative side bearing. Journal of Guidance, Control and Dynamics, 2016, 39(6): $1454-1460$.

[9] LI Z Y, HOVAKIMYAN N, DOBROKHODOV V, et al. Vision-based target tracking and motion estimation using a small UAV. Proc. of the 49th IEEE Conference on Decision and Control, 2010: 2505-2510.

[10] LAWREANCE W, FREW E, PISANO W. Lyapunov vector field for autonomous UAV flight control. Proc. of the AIAA Guidance and Navigation Control Conference, 2007: $6317-$ 6339.

[11] NELON D R, BARBER D B, MCLAIN T W, et al. Vector field path following for miniature vehicles. IEEE Trans. on Robotics, 2007, 23(3): 519-529.

[12] NIKI R, MATTEO Z. 2D tracking and over-flight of a target by means of a non-linear guidance law for UAV. Proc. of the IEEE Aerospace Conference, 2009: 1-11.

[13] NIKI R, MATTEO Z. Fixed-wing UAV guidance law for surface-target tracking and overflight. Proc. of the IEEE Aerospace Conference, 2012: 1-11.

[14] REGINA N, ZANZI M. A novel solution for overflight and surveying of a collaborative target with fixed wing UAV. Proc. of the AIAA Guidance, Navigation, and Control Conference, 2012: $1627-1652$.

[15] OH H, KIM S, SHIN H S, et al. Rendezvous and standoff target tracking guidance using differential geometry. Journal of Intelligent \& Robotic Systems, 2013, 69(1-4): 389-405.

[16] HYONDONG. Towards autonomous surveyllance and tracking by multiple UAVs. Oxfordshire, England: Cranfield University, 2013.

[17] ZHANG M, XIA W, CHEN X. A unitized guidance law for over-flight and standoff tracking of a ground target with fixed wing unmanned aerial vehicle. Proc. of the Institution of Mechanical Engineers, Part G: Journal of Aerospace Engineering, 2018, 232(6): 1024-1034.

[18] ZHANG M, TIAN P F, CHEN X. UAV guidance law for circumnavigation a ground target and stability proof. Acta Aeronautica et Astronautica Sinica, 2016, 37(11): 3425-3434. (in Chinese)

[19] OH H, KIM S, SHIN H S, et al. Coordinated standoff tracking of moving targets groups using multiple UAVs. IEEE Trans. on Aerospace and Electronic Systems, 2015, 51(2): $1501-1514$.

[20] POTHEN A A, RATNOO A. Curvature-constrained Lyapunov vector field for standoff target tracking. Journal of Guidance, Control and Dynamics, 2017, 40(10): 1-11.

[21] OH H, TURCHI D, KIM S, et al. Coordinated standoff tracking using path shaping for multiple UAVs. IEEE Trans. on Aerospace and Electronic Systems, 2014, 50(1): 348-363.

[22] MRUDULA A P, NANDAGOPAL J L. Line-of-sight guidance for formation control of quadrotor. Proc. of the International Conference on Circuit, Power and Computing Technologies, 2017: $1-8$.

[23] NIKI R, MATTEO Z. UAV guidance law for ground-based target trajectory tracking and loitering. Proc. of the Aerospace Conference, 2011: 1-9.
[24] ZHANG M, LIU H T. Cooperative tracking a moving target using multiple fixed-wing UAVs. Journal of Intelligent \& Robotic Systems, 2016, 81(3/4): $505-529$.

[25] ZHANG M, TIAN P, CHEN X, et al. Ground target tracking guidance law for fixed-wing unmanned aerial vehicle: a search and capture approach. Journal of Dynamic Systems, Measurement, and Control, 2017, 139(10): 104503.

[26] MODIRROUSTA A, SOHRAB M, DEHGHAN S M M. A modified guidance law for ground moving target tracking with a class of the fast adaptive second-order sliding mode. Transactions of the Institute of Measurement \& Control, 2016, 38(7): $1-13$.

[27] WU K, CAI Z, ZHAO J, et al. Target tracking based on a nonsingular fast terminal sliding mode guidance law by fixed-wing UAV. Applied Sciences, 2017, 7(4): 1-18.

[28] SUN L, PACK D. Guidance law design for tracking mobile ground targets using an unmanned aerial vehicle with a fixed camera. Proc. of the International Conference on Unmanned Aircraft Systems, 2016: $235-241$.

[29] MODIRROUSTA A, SOHRAB M, DEHGHAN S M M. A modified guidance law for ground moving target tracking with a class of the fast adaptive second-order sliding mode. Transactions of the Institute of Measurement \& Control, 2016, 38(7): $1-13$.

[30] JUNG Y, CHO S, SHIM D H. A trajectory-tracking controller design using L1 adaptive control for multi-rotor UAVs. Proc. of the International Conference on Unmanned Aircraft Systems, 2015, 42(10): $132-138$.

[31] WU S T, FEI Y H. Flight control system. Beijing: Beihang University Press, 2005. (in Chinese)

[32] KHALIL H K. Nonlinear Systems. 3rd ed. Upper Saddle River: Prentice Hall, 2002.

[33] CHOI H, KIM Y. UAV guidance using a monocular-vision sensor for aerial target tracking. Control Engineering Practice, 2014, 22(1): $10-19$.

[34] XIONG J, NIU Y. Guidance law for multi-UAVs collaborative ground target tracking under obstacle environment. Proc. of the Chinese Control and Decision Conference, 2017: 7219-7223.

[35] WU Z, GUAN Z, YANG C, et al. Terminal guidance law for UAV based on receding horizon control strategy. Complexity, 2017, 2017(6): 1-19.

\section{Biographies}

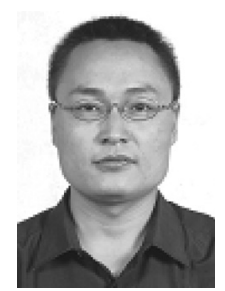

ZHANG Min was born in 1973. He received his M.S. degree and Ph.D. degree in navigation guidance and control from Nanjing University of Aeronautics and Astronautics. Now, he is a professor in College of Automation Engineering, Nanjing University of Aeronautics and Astronautics. His research interests include flight control, robust control and nonlinear optimization algorithm.

E-mail: zhangmin@nuaa.edu.cn

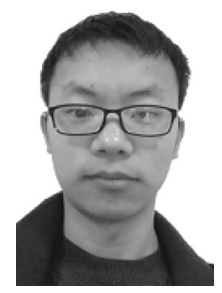

ZHENG Chenming was born in 1993. He received his B.S. degree in the College of Information Science and Engineering from Nanjing University of Aeronautics and Astronautics. He is currently an M.S. student in the College of Automation Engineering from Nanjing University of Aeronautics and Astronautics. His research interests include flight control, and nonlinear control system. E-mail: 605520339@qq.com 


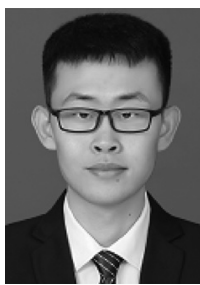

HUANG Kun was born in 1993. He received his B.S. degree in the College of Information Science and Engineering from Nanjing University of Aeronautics and Astronautics. He is currently an M.S. student in the College of Automation Engineering from Nanjing University of Aeronautics and Astronautics. His research interests include flight control, nonlinear control system, etc.

E-mail: 2275087646@qq.com 